MATEC Web of Conferences 6, 05009 (2013)

DOI: $10.1051 /$ matecconf/20130605009

(C) Owned by the authors, published by EDP Sciences, 2013

\title{
Simplified stochastic modeling of concrete spalling due to fire
}

\author{
I.J.J. van Straalen ${ }^{1}$, R.D.J.M. Steenbergen ${ }^{1}$, S.S.K. Lentzen ${ }^{1}$ and R. de Vries ${ }^{2}$ \\ 1 TNO, Delft, The Netherlands \\ 2 Arup, Amsterdam, The Netherlands
}

\begin{abstract}
Predicting spalling of concrete due to fire loading is undoubtedly a complex task to come across. Existing numerical models are dealing with the phenomena on different complexity levels of describing the physical/chemical processes and material behavior. But still they do not take the highly stochastic nature of these processes into account. This paper discusses a proposal to develop a practical prediction method based on simplify numerical models and the probabilistic approach.
\end{abstract}

\section{INTRODUCTION}

For daily practice, the Eurocode EN 1992-1-2 [1] provides general boundary conditions for the material properties of concrete and steel reinforcement that vary with increasing temperatures. It also provides detailed and simplified methodologies for the design of concrete structures under fire loading. Regarding spalling, the Eurocode requires that (explosive) spalling has to be avoided by appropriate measures, or as an alternative, its effects on the performance requirements have to be taken into account. As a design rule it describes that explosive spalling is unlikely to occur when the moisture content of the concrete is less than $\mathrm{k} \%$ by weight. In the national annex for the Netherlands, the k-value is assumed to be equal to 3 . In case of the alternative, the reduction of the load bearing capacity and/or separation function due to spalling necessitates a risk assessment. A more elaborated review on European and national standards and guidelines can be found in [2].

To predict spalling of concrete more realistically, it is important to find a spalling prediction method which balances the required detail in theoretical accuracy and the practical applicability. In literature numerous attempts can be found to model spalling on a micro-, a meso- and a macro-mechanical level. Besides the disadvantage that the proposed simplified models often leads to thermodynamic inconsistencies, the models also ignore the highly stochastics processes involved. So far the right balance has not been found.

Within this paper the results of a literature review of numerical models developed are presented and discussed. In addition attention is paid towards the stochastic nature of spalling of concrete and it is explained how the probabilistic approach might be applied to take this nature into account. Based on this knowledge a procedure is proposed how to simplify finite element models and how to develop a stochastic version. Finally a proposal is made to develop a practical prediction method based on such simplified stochastic modeling of spalling of concrete due to fire loading.

\section{NUMERICAL MODELS}

\subsection{Underlying physics and chemistry}

In order to predict spalling of concrete, it is important to understand the underlying mechanisms and the media in which these mechanisms occur. The mechanisms emerge from physical and chemical processes

This is an Open Access article distributed under the terms of the Creative Commons Attribution License 2.0, which permits unrestricted use, distribution, and reproduction in any medium, provided the original work is properly cited. 


\section{MATEC Web of Conferences}

Table 1. Transport mechanisms in concrete.

\begin{tabular}{|c|c|c|}
\hline \multicolumn{2}{|l|}{ Transport phenomena } & Physical mechanisms \\
\hline \multicolumn{2}{|l|}{ Heat flow } & $\begin{array}{l}\text { Heat conduction and convective transport of } \\
\text { energy (with flowing fluids - gas and water) within } \\
\text { the pores of solid. Heat exchange by convection } \\
\text { and radiation at external surfaces }\end{array}$ \\
\hline \multicolumn{2}{|c|}{ Solid mass transfer (mechanical deformation) } & $\begin{array}{l}\text { Mechanical displacement (due to thermo-hydro- } \\
\text { mechanical loading) }\end{array}$ \\
\hline \multirow[t]{3}{*}{ Liquid mass transfer } & Capillary water & Darcian flow (due to water pressure gradient) \\
\hline & Adsorbed water & $\begin{array}{l}\text { Diffusional Fick's flow (due to saturation degree } \\
\text { gradient) }\end{array}$ \\
\hline & Chemically bound water & No fluid transport (part of the solid matrix) \\
\hline \multirow[t]{2}{*}{ Gas mass transfer } & Dry air & $\begin{array}{l}\text { Darcian flow (due to gas pressure gradient) } \\
\text { and diffusional Fick's flow (caused by dray air } \\
\text { concentration gradient) }\end{array}$ \\
\hline & Water vapour & $\begin{array}{l}\text { Darcian flow (due to gas pressure gradient) and } \\
\text { diffusional Fick's flow (caused by water vapour } \\
\text { concentration gradient, influenced by the Knudsen } \\
\text { effect in narrow pores) }\end{array}$ \\
\hline
\end{tabular}

occurring in heated concrete. Besides the transportation of energy, the physically processes consist of mass being transported in either the gas, the liquid or the solid state. Besides mechanical forces also chemical and other phase changing processes are driving factors of the transport phenomena. Therefore the underlying processes of spalling as described in literature [3-7], are briefly addressed by describing the transport phenomena and the occurring phase change processes.

Concrete is a porous medium consisting of a solid skeleton, and pores filled either with a liquid medium (capillary water and physically absorbed water) or with a gaseous medium (moist air). Water also exists in chemically bound form, and this phase is attributed to the solid skeleton. All three media (solid, liquid and gas) obey the laws of mass balance. Consequently, transport phenomena can be identified which facilitate fulfillment of the balance laws. A summary of transport phenomena and matching physical mechanisms is given in Table 1 .

Phase change processes take place, which can be physical or chemical in nature. For Portland cement based concrete they are: $\mathrm{C}-\mathrm{S}-\mathrm{H}$ (cement gel) and $\mathrm{CH}$ dehydration/hydration, evaporation/condensation, sorption/desorption, aggregate physical phase changes (e.g. $\alpha-\beta$ transformation of quartz at $573{ }^{\circ} \mathrm{C}$ ), and decarbonation of calcium carbonate above $650^{\circ} \mathrm{C}$. A summary of the phase change processes is given in Table 2. The phase changing processes are additionally depicted in Figure 1. It is noted that all these phase changes are strongly influenced by temperature, and chemical ones are thermodynamically not fully reversible, although reversibility is often assumed in the present models.

\subsection{Review of models}

As summarized in 2.1, the underlying mechanisms causing spalling in concrete, are subdivided into transport phenomena of media in different phases and into phases changing processes. Both are nonlinearly coupled. The transport mechanisms are generally described using continuum mechanical principles (balance equations) in a Lagrangean setting (fixed reference configuration). As the number of balance equations is too small to solve all unknown quantities, it is necessary to choose dependent (e.g. stress or heat flux) and independent quantities (e.g. strain or temperature) which are subsequently coupled using additional or constitutive relations (e.g. Hooke's or Fourier's law). It is therefore natural to take the phase changing processes into consideration in the constitutive part of the models. 
Table 2. Summary of phase changes in concrete.

\begin{tabular}{|c|c|c|c|}
\hline Phase & Process & Energy & Phase change process \\
\hline Dehydration & \multirow[t]{2}{*}{ Chemical } & Loss & $\begin{array}{l}\text { Solid matrix }(\mathrm{CSH} \text { or } \mathrm{CH}) \rightarrow \text { water }- \text { energy }+ \text { matter }(\mathrm{C} \text { or } \\
\left.\mathrm{C}_{x} \mathrm{~S}\right)\end{array}$ \\
\hline Hydration & & Gain & $\begin{array}{l}\left.\text { Water }+ \text { matter }\left(\mathrm{C} \text { or } \mathrm{C}_{x} \mathrm{~S}\right)\right) \rightarrow \text { solid matrix }(\mathrm{CSH} \text { or } \mathrm{CH}) \\
+ \text { energy }\end{array}$ \\
\hline Evaporation & \multirow{2}{*}{ Physical } & Loss & Capillary water $\rightarrow$ water vapour - energy \\
\hline Condensation & & Gain & Water vapour $\rightarrow$ capillary water + energy \\
\hline Desorption & \multirow{2}{*}{ Physical } & Loss & Physically adsorbed water $\rightarrow$ water vapour - energy \\
\hline Sorption & & Gain & Water vapour $\rightarrow$ physically adsorbed water + energy \\
\hline Decarbonation & \multirow{2}{*}{ Chemical } & Loss & Calcium carbonate $\rightarrow \mathrm{CO}_{2}+$ matter $(\mathrm{CaO})$ - energy \\
\hline Carbonation & & Gain & $\mathrm{CO}_{2}+$ matter $(\mathrm{CaO}) \rightarrow$ Calcium carbonate + energy \\
\hline$\alpha-\beta$ inversion & \multirow{2}{*}{ Physical } & Loss & Endothermic during heating up at $573^{\circ} \mathrm{C}$ with expansion \\
\hline$\beta-\alpha$ inversion & & Gain & Exothermic during cooling down at $573^{\circ} \mathrm{C}$ with contraction \\
\hline
\end{tabular}

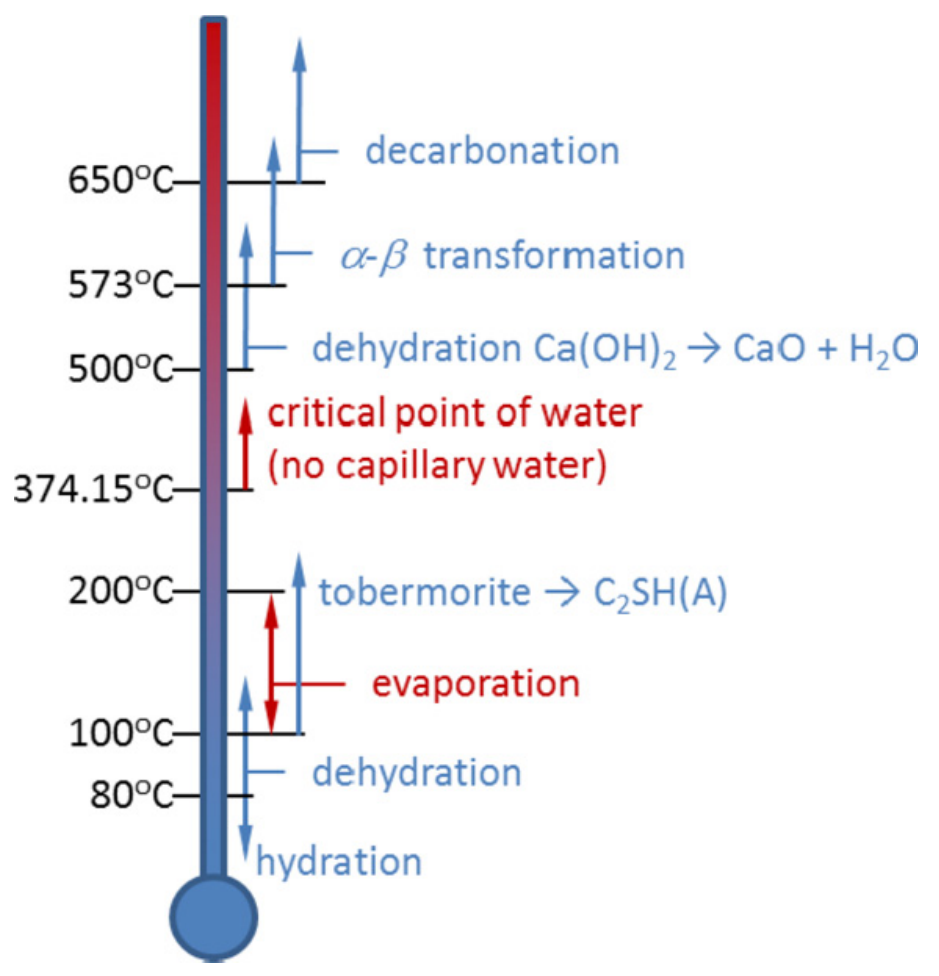

Figure 1. Temperature scale with phase changing processes.

Generally, the continuum mechanics of spalling of concrete under fire load can be treated on three different scales, namely on the micro-, meso- and macro-scale. At the microscale the concrete is represented by three types of solid (aggregate, solid skeleton paste in two forms - bond region and matrix) and separate pores through which fluids flow. The pores, microcracks, aggregate and skeleton are explicitly modeled in the sense that these micro-structures are visually perceptible in the model. Micro-models are meant to co-exist next to measurements to feed the meso- and macro-models with material parameters [8]. For all the phases and media in the concrete the balance laws are solved in 


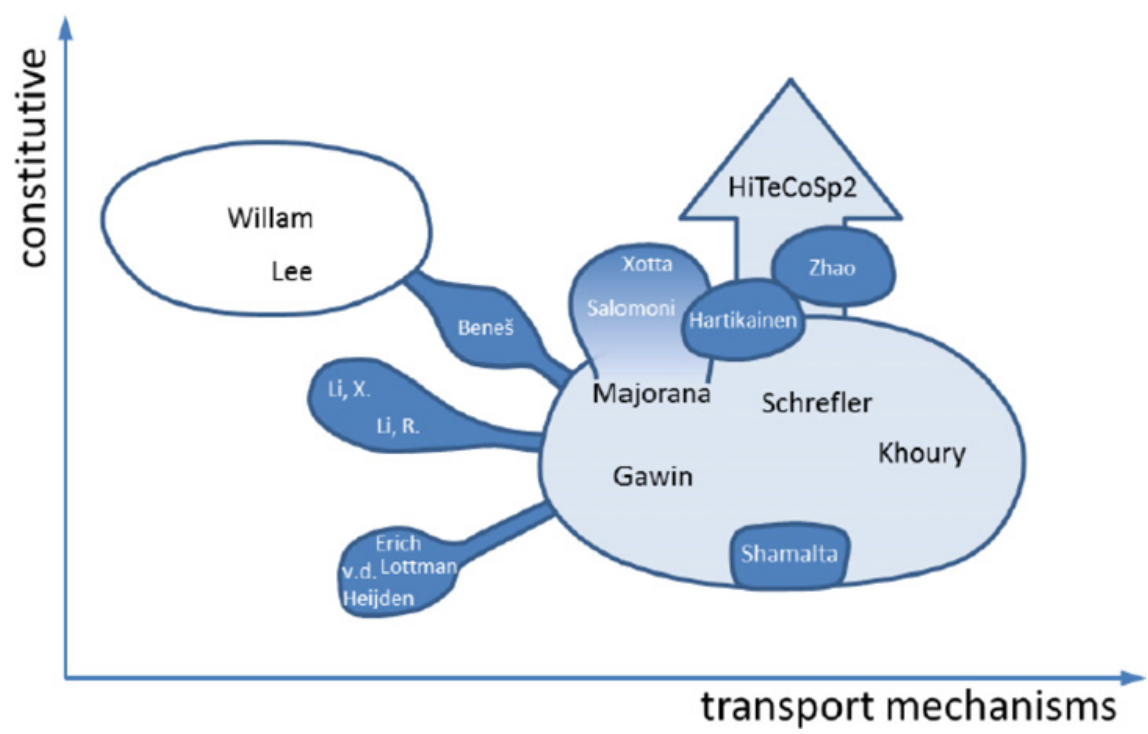

Figure 2. Identified research groups in the field of modeling the spalling phenomenon.

the micro-scale models. In the meso- and the macro-models the microstructures are smeared within a representative element volume. At the meso-level, concrete is represented by aggregate, bond region and paste/mortar with separate characteristics for each domain. At the macro-level even the three types of regions are smeared into one mean material.

When such high complexity problems are to be modeled it is common practice to build the models according to the eight fundamental axioms of continuum mechanics [9]: causality, determinism, equipresence, neighbourhood, memory, objectivity, material invariance and admissibility. Some of these axioms do not require special attention and are very likely to be fulfilled without effort, while others play an important role when building the models describing spalling of concrete under fire loading.

Schrefler, Gawin, Majorana and Pesavento is a group of researchers who has made an important contribution to thermo-hydro-chemo-mechanical models to described the spalling behavior in concrete [3, 10-14]. These models are created without dropping any quantity, physical process or constitutive coupling. This leads for instance to models based on material properties which cannot be fully measured yet (e.g. strength at elevated temperatures). Therefore a vast amount of literature (more than 25 references have been identified by the authors) can be seen as a simplification of the complex models, excluding the irrelevant phenomena and circumventing the processes which cannot be characterized yet.

The identified research groups in the field of modeling the spalling phenomenon are displayed in Figure 2. The resulting models of each research group are assessed with respect to the completeness/complexity of the transport mechanisms and the constitutive assumptions. Additionally, an attempt is made to depict the relations between the research groups. It can be concluded that none of the available models given in literature cover the third quadrant in which completeness is strived in the representation of the transport phenomena as well as the constitutive behaviour.

From continuum mechanics point of view, the research conducted in the BRITE EURAM III project HiTeCo [15] is fundamentally most sound. However, it lacks in the sufficient description of certain constitutive aspects concerning failure, namely:

- A damage parameter D is introduced which constitutes mechanical, thermal and thermo-chemical damage. No quantitative relation is given between this parameter and the actual occurrence of spalling.

- In explosive spalling buckling effects play an important role. These have not been considered. 
IWCS 2013

- The material deterioration during the cooling period has not been taken into account. In order to overcome these drawbacks, the HiTeCoSp tool developed in [15] has been extended with new features in the research of the UPTUN-project [16] and the resulting tool is referred to as HiTeCoSp2. This development has also been illustrated in Figure 1.

\subsection{Discussion of models}

The reviewed models describing the processes in heated concrete are based on continuum mechanics and due to their complexity the solutions are approximated using numerical procedures. In most cases a Finite Element Method (FEM) is applied. At present the models developed in the UPTUN-project [16] are the most elaborate ones. However, the numerical implementation is only applicable to studying spalling of concrete in tunnels. Another drawback is the amount of (difficult/impossible to determine) input (material) parameters. The tool HITECOSP2 is equipped with a material database containing the required material parameters of a set of materials. Judging from ongoing research [17] it appears to be still a challenge to feed the model with the appropriate input data. Finally, all developed models are deterministic in nature. Deterministic models deliver spurious answers when applied to highly stochastic problems such as spalling of concrete.

\section{STOCHASTIC MODELS}

\subsection{Impact of stochastic behaviour}

Experiments intended for validation of the spalling prediction methods show that the results suffer from stochastic effects. Although the initial conditions (as for instance cylinder spalling tests) such as preloading, moisture content and aggregate ratio are apparently the same, the results between different laboratories and even within one test programme, vary substantially. Also experiments to determine the value of the input parameters of models show large scatter bands. The strong influence of the stochastic behavior of the spalling process underlines the opinion that deterministic prediction methods will not result into adequate predictions. This underline the necessity to create prediction tools based on probabilistic approaches.

\subsection{Probabilistic approach}

The principles of the probabilistic approach can be traced back to the risk-analysis. Probabilistic techniques calculate the possibility that a certain occasion occurs. To do this, models that simulate reality should be used. Instead of using one set of deterministic input parameters, within the probabilistic approach the simulation model can handle a set of stochastic input parameters. One of the simplest probabilistic techniques is Monto Carlo. With this technique thousands of simulations are carried out, each time with a different set of input parameters taken randomly from their own stochastic distributions. The results of those simulations give the probability that the occasion (like spalling of concrete) occurs. This probability of the event considered has to be compared with the requirements as stated by e.g. the Eurocode [18].

The probabilistic approach is based on mathematics, with its basis into probability and statistics. Within probabilistic techniques the statistical descriptions are represented by the distributions of the input parameters and the correlations between those parameters. The assessment of the properties of these distributions and correlations is an active topic of research. Remarkable efforts have been collected in the Probabilistic Model Code [19]. With use of the probabilistic approach complete systems can be analyzed, including time dependent phenomena. Some of the available probabilistic techniques make it possible to discriminate which of the stochastic parameters is dominant and which is not. 


\section{MATEC Web of Conferences}

To make probabilistic techniques available within the field of research regarding spalling of concrete, the following issues have to be considered:

- The requirements stated by the Eurocode [18] do not give any guidance which value has to be taken for the required probability that spalling of concrete might occurs. To determine this value it is necessary to perform a risk-analyses on basis of realistic scenarios of structural failure due to spalling.

- Probabilistic techniques have to be combined with an appropriate simulation model. As discussed in section 2.3, a FEM model seems to be the most promising one.

\subsection{Stochastic finite element models}

Examining literature on the subject of using FEM as the simulation model within the stochastic context, reveals the existence of two methods:

- Unintrusive methods that utilize the deterministic FEM software in a stochastic framework. Unintrusive methods are considered to be well-developed and is commonly based on Monte Carlo simulation combined with various efficiency improvement strategies. Unintrusive methods utilize existing deterministic FEM software in a stochastic framework, commonly based on Monte Carlo simulation combined with various efficiency improvement strategies (such as surrogate limit state functions, advanced sampling techniques, etc.). A disadvantage of the unintrusive methods might be the curse of dimensionality in case of a large number of stochastic parameters. It can be considered to be a well-understood field of science; a good reference on the subject is [20].

- Intrusive methods that require a specialized stochastic FEM code, but hold the promise of being much more efficient. Intrusive methods have gained less attention in literature [21]. The theoretical groundwork is there, but applications to real-world/large-scale problems is lacking. Intrusive methods are specialized versions of the finite element method where the stochastic character of parameters is accounted for in the mathematical formulation. By taking into account the stochastic character of parameters from the start, the methods hold the promise of being much more efficient. This property is confirmed in literature, although depending on the application and only for small-scale problems. Applications to real-world/large-scale problems are lacking and need more research.

\section{PROPOSAL TO DEVELOP A PRACTICAL PREDICTION METHOD}

To develop a practical prediction method for spalling of concrete, a bifurcation from the academic path of developing more complex models is suggested. The models developed in the UPTUN project are a good starting point for the practical path. The next steps along this path consist of studying the possibilities to:

- simplify the UPTUN-models and to make them applicable to concrete structures other than tunnels,

- incorporate the probabilistic approach into the numerical procedures, and

- tailor the (laboratory) experiments and expert opinions to facilitate the practical path to predict spalling.

To apply such a practical spalling prediction method as an engineering tool, the following issues should be taken into account:

- Easily obtained input data. The main requirement to get easily obtained input implicates that it is technically feasible to acquire data for reasonable costs. Since the costs of an extended fire test programme are too high and it is for instance not (yet) possible to measure the temperature and stress dependent coupling factors between the saturation level and the permeability, a prediction method should be based on the statistical coupling of global measurements, expert judgement and simplified numerical methods.

- Numerically stable method. Numerical stability is required on the fact that the engineer is not necessarily a numerical expert. For instance the large pressure gradients at the vapour fronts in the material can cause instabilities. The results should converge steadily with increasing mesh resolutions. 
The term mesh resolution is to be interpreted broadly; it does not necessarily refer to the numerical discretization, but it can for instance also refer to the amount of test data on which the prediction is based.

- Reproducible results. The reproducibility requirement is a logical one, but it is often an unaddressed issue. At the micro- and mesoscale this requirement is very difficult to be fulfilled, while this is mostly no issue when predicting on the macroscale. However, these conditions are rarely given. The preferred outcome of the practical prediction method is a choice between whether or not to protect the concrete against fire. This kind of outcome is binary in nature and it endangers the fulfillment of the reproducibility requirement. If the preferred outcome is accompanied with a statistically-based risk analysis, the reproducibility requirement is more easily met and the engineers are provided with the appropriate outcome to make the right decisions.

- Reasonable computing times. According to the convergence requirement (numerical stability) one could conclude that more accurate tools required more computation time. This means that computational efficiency is of great importance.

\section{CONCLUSION}

From an academic point of view it is natural to strive for spalling prediction by using continuum mechanical models in combination with finite element methods. The state-of-the-art, as achieved in the UPTUN project [16], is from that point of view, heading towards the ultimate goal. It is however important to keep in mind that such a prediction method might not be practical, understandable, reproducible and therefore also not reliable enough for the engineering practice. A translation from the academic to the practical prediction approach is required.

Since the spalling process is highly stochastic, the deterministic model should be enriched by the probabilistic approach. Using FEM as the simulation model within the stochastic context needs further research in finding the efficient and effective methods. The probabilistic approach also require new stochastic input data from tests, combined with expert judgement. Additionally research is needed to set the required probability of spalling of concrete.

\section{References}

[1] EN 1992-1-2, Eurocode 2: Design of concrete structures - Part 1-2: General rules - structural fire design, CEN, 2004.

[2] Schulte A., Schaab A., Fire protection requirements and solutions for international tunnel projects, Concrete spalling due to fire exposure, Proc. of the 2nd international RILEM workshop, pages 3-17, 2011.

[3] Schrefler B.A., Khoury G.A., Gawin D., Majorana C.E., Thermo-hydromechanical modelling of high performance concrete at high temperatures, Eng. Computation, 19(7), 787-819, 2002.

[4] Chaube R.P., Shimomura T., MaekawaK., Multi-phase water movement in concrete as a multicomponent system, in Bažant Z.P. and Carol I., editors, Fifth RILEM International Symposium on Creep and Shrinkage of Concrete, 1993.

[5] Verbeck G., Copeland L.E., Some physical and chemical aspects of high pressure steam curing, ACI publication SP, 32(1),1-13, 1972.

[6] Harmathy T.Z., Thermal properties of concrete at elevated temperatures, J. ASTM Materials, 5(1), 1970.

[7] Khoury G.A., Sullican P.J.E., Grainger B.N., Transient thermal strain of concrete literature review, conditions within specimen and individual constituent behavior, Mag. of Concr. Research, 37(132), 131-144, 1985.

[8] Zhang Q., Microstructure and deterioration mechanisms of Portland cement at elevated temperature, $\mathrm{PhD}$ thesis, Technical University Delft, 2013. 


\section{MATEC Web of Conferences}

[9] Eringen A.C., Nonlocal field theories. Springer-Verlag, New York, 2002.

[10] Gawin D., Majorana C.E., Schrefler B.A., Numerical analysis of hygrothermal behaviour and damage of concrete at high temperature, Mech. Cohes.-Fric. Mater., 4, 37-74, 1999.

[11] Gawin D., Pesavento F., Schrefler B.A., Modelling of hygro-thermal behavior and damage of concrete at temperature above the critical point of water, Int. J. Numer. Anal. Meth. Geomech., 26, 537-562, 2002.

[12] Gawin D., Pesavento F., Schrefler B.A., Modelling of hygro-thermal behavior of concrete at high temperature with thermo-chemical and mechanical material degradation, Comp. Meth. Appl. Mech. Eng., 192,1731-1771, 2003.

[13] Gawin D., Pesavento F., Schrefler B.A., Modelling damage processes of concrete at high temperature with thermodynamics of multi-phase porous media, J. Theor. Appl. Mech., 44(3), 505-532, 2006.

[14] Schrefler B.A., Pesavento F., Gawin D., A multiphase model for concrete: numerical solutions and applications, Proc. of the 2nd WSEAS Int. Conference on Applied and Theoretical Mechanics, Venice, Italy, 108-116, 2006.

[15] Brite Euram III BRPR-CT95-0065 HITECO, Understanding and industrial application of high performance concrete in high temperature environment, 1999.

[16] Corsi F., Schrefler B.A., Majorana C., Pesavento F., Gawin D., Workpackage 4 Fire effects and tunnel performance: system structural response, m42: Qualified and validated procedure for spalling evaluation tunnels, Technical Report FP5 Contract G1RD-CT-2002-766, EU, 2008.

[17] Koenders E.A.B., Dehn F., Concrete spalling due to fire exposure, Rilem publications S.A.R.L., 2011.

[18] EN 1990, Eurocode: Basis of structural design, CEN, 2002.

[19] Joint Committee on Structural Safety, Probabilistic Model Code, 2007.

[20] Waarts, P.H., Structural reliability using Finite Element Analysis, an appraisal of DARS, PhD thesis, Delft University of Technology, 2000.

[21] Vries R.de, The Stochastic Finite Element Method (SFEM) - Intrusive formulations and an application in Structural Dynamics, Master thesis, Technical University Delft, 2013. 